\title{
Individualized ablation strategy guided by live simultaneous global mapping to treat persistent atrial fibrillation
}

\author{
Rui Shi ${ }^{1}$, Mark Norman ${ }^{1}$, Zhong Chen ${ }^{1}$ \& Tom Wong*,1 \\ ${ }^{1}$ Heart Rhythm Center, The Royal Brompton \& Harefield NHS Foundation Trust, National Heart \& Lung Institute, Imperial College \\ London, London, UK \\ *Author for correspondence: Tel.: +44 207351 8619; t.wong2@rbht.nhs.uk
}

\begin{abstract}
Atrial fibrillation (AF) is the most common clinical arrhythmia encountered. Catheter ablation has become the first-line therapy for symptomatic drug-refractory paroxysmal and persistent AF. Although pulmonary vein electrical isolation is still the cornerstone of the ablation strategy, the clinical outcome particularly in treating persistent AF is suboptimal. Significant efforts have been applied with live global chamber mapping of AF aimed to identify patient-specific drivers and/or maintainers located outside of the pulmonary veins to further improve the outcome of catheter ablation. Within this review, we present an overview of contemporary global chamber AF mapping technologies and characteristics, with a particular focus on global, noncontact, dipole density mapping illustrated with a clinical case of persistent AF ablation using this novel methodology.
\end{abstract}

First draft submitted: 19 December 2017; Accepted for publication: 1 February 2018; Published online: 14 February 2018

Keywords: atrial fibrillation $\bullet$ catheter ablation $\bullet$ dipole density noncontact mapping

Atrial fibrillation (AF) is the most common arrhythmia encountered in clinical practice, affecting approximately $1.5-2 \%$ of the general population $[1,2]$. The prevalence of $\mathrm{AF}$ will continue to increase as the population ages [3-6]. The persistent and permanent forms of AF are the most frequent, representing $68-80 \%$ of patients identified of the general population or in patients hospitalized for AF, and with approximately $20 \%$ having suffered at least two recurrences during the previous year of observation [7-11]. Moreover, AF is far from benign, it incurs a fivefold increased risk of stroke [12,13], threefold increased risk of heart failure [14], twofold increased risk of mortality [15], 2.4-fold increased risk of development of dementia [16,17] and even sudden death [18,19]. Technical advancements in catheter-based intervention over the past decades has increased the application of catheter ablation of AF, with ablation often chosen as the first-line treatment for symptomatic drug-refractory paroxysmal and persistent $\mathrm{AF}$ [20-22] over an exclusively pharmacological treatment [23-26].

\section{Evolution of AF ablation \& the challenges in ablation of persistent AF}

The mechanisms of AF are not well understood despite intense investigations at least over the past century. Three main hypotheses concerning the mechanisms of AF include multiple re-entrant wavelets, rapidly discharging automatic foci and a single re-entrant circuit with fibrillatory conduction [27-29]. A key breakthrough was the recognition of rapidly firing foci which triggered $\mathrm{AF}[30,31]$. This discovery ushered in the modern era of performing pulmonary vein electrical isolation (PVI) for primarily treating paroxysmal $\mathrm{AF}[31,32]$ with its application subsequently extended to persistent $\mathrm{AF}$ as well [32-34].

Currently, PVI is the cornerstone of ablation for both paroxysmal and persistent AF, although the clinical outcome of PVI alone to treat persistent AF is not satisfactory [34-36]. Consequently, adjunctive ablation strategies in addition to PVI have been investigated, which included targeting the complex fractionated atrial electrograms [37,38], linear ablation [39-42], ganglionated plexi ablation [43-45], nonpulmonary vein (PV) triggers [46-48], left atrial appendage electrical isolation [49,50] and so on. Despite encouraging acute outcomes of ablating persistent AF in some studies, the medium to longer term follow-up clinical outcomes were less impressive [39,51-53].

Future Medicine 
The challenges to the success in ablating persistent AF [54] include: the lack of full understanding of the mechanisms of AF specific to the individual patient being treated, although there is a broad acceptance of the concept that triggers and substrate are essential to the initiation and the maintenance of AF [55-57]; the limited ability to identify non-PV atrial triggers [58] (identified in only 10-33\% of unselected AF patients referred for catheter ablation) on the posterior left atrial wall, the superior vena cava, the crista terminalis, the coronary sinus, the ligament of Marshall and the left atrial appendage [49,59-62]; and difficult to achieve complete transmural and durable lesion from endocardial ablative approach, although the use of contact force-sensing technology [63-67], adenosine testing after ablation [68-70] and pace capture-guided ablation [71] may be of help.

\section{Global mapping of AF using current technologies}

Noncontact endocardial mapping with EnSite ${ }^{\circledR}$ Array

Due to the complex mechanisms and irregular activation of AF, conventional point-by-point mapping may be difficult or even not feasible. Thus, the first noncontact, global chamber mapping technology (EnSite Array, Abbott/St. Jude Medical, Inc., MN, USA) emerged early on in the history of 3D mapping, both in terms of technology and concept in mapping AF [72]. The multipolar noncontact catheter has 64 unipolar electrodes, which record cavitary unipolar electrograms and calculate endocardial unipolar electrograms using a mathematical inverse algorithm, which is a solution to Laplace's equation. The calculated electrograms are displayed on a surface mesh with 2592 rectangular facets. The mesh represents the geometry of the endocardial surface that is physically traced by a conventional catheter [73].

There are limitations of using this technology in mapping AF, which include: the precision of the inversely calculated electrograms (EGMs) depended on the distance from the center of the array to the endocardial surface, and the method was validated only for distances $<40 \mathrm{~mm}$ [73,74]; it is not consistently used for catheter ablation of $\mathrm{AF}$, although it may facilitate recognition of gaps in ablation lines and for localization of arrhythmogenic non-PV foci [72]; and the difficulties of manipulating ablation catheter with the balloon mapping catheter in the atrial chamber, may have restricted the widespread use of this technology.

\section{Contact focal impulse \& rotor modulation phase mapping}

The Topera RhythmView ${ }^{T M}$ 3D Mapping System (Abbott/Topera Medical, CA, USA) utilizes the FIRMap ${ }^{\text {TM }}$ Catheter, a 64-electrode, contact mapping basket catheter, which collects electrophysiologic activity, and translates this to an existing geometry, that is created on a non-Topera 3-D mapping system of the atrial chamber [75]. Proprietary software processes electrograms through a phase mapping algorithm with analysis of repolarization and conduction dynamics to identify electrical sources of AF rotors and foci, and thereby guide focal impulse and rotor modulation (FIRM) ablation [76]. The technology identified sites of spiral rotors and focal drivers of AF in humans for the first time without the need for incorporating surgically placed electrodes. In individual FIRM guided ablation studies, a wide variation in mid-term and long-term freedom from AF (17-80\%) has been reported [76-82].

Within the variation of results from the various case series of FIRM mapping, it has been postulated that perhaps some of the differences are due to the catheter shape resulting in unequal topographical coverage of the atria and in some cases electrodes either not being in contact (46\%) [80], catheter protruding through the mitral valve or electrogram quality was unusable. From groups with both high and low result success in terms of outcome, the conclusion was that a multicenter trial of FIRM mapping would be useful. In the first small multicenter trial of FIRM mapping involving 78 patients at ten experienced centers, freedom from AF at 1 year was reported as $80 \%[83]$.

Although cardiac electrophysiologic activity of the chamber could be acquired simultaneously, limitations remain with this methodology [84]. First, the electrode contact on the atrial wall is not consistent, leading to inadequate electrogram resolution required for rotor site identification. Second, localization of contact catheters to guide an ablation catheter to the FIRM map target is absent.

\section{Noncontact body surface mapping}

The ECVUE ${ }^{T M}$ Mapping System (CardioInsight, Medtronic, MN, USA), is a noninvasive body surface mapping system that uses 252 external electrodes in combination with computed tomography to record bi-atrial unipolar electrograms and create simultaneous bi-atrial 3D maps [85-87]. Proprietary body-surface phase mapping algorithms are applied to the selected AF segment to identify active AF driver regions (classified as focal or re-entrant) [88,89]. 


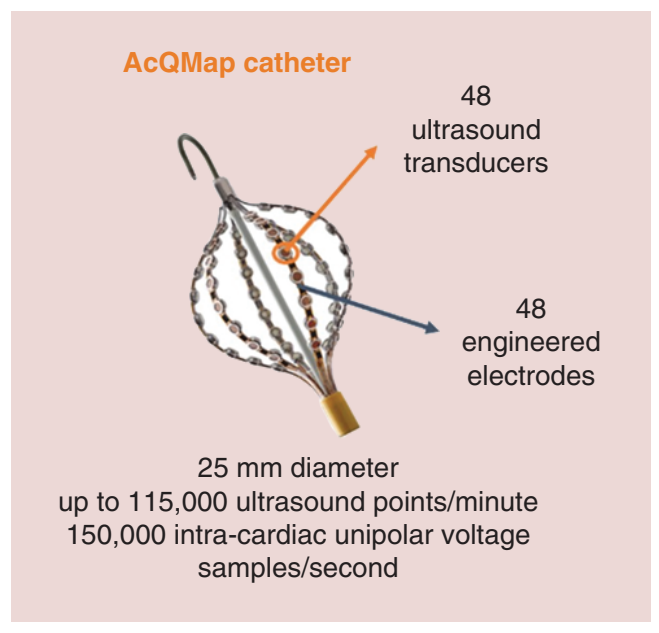

Figure 1. The ACQMap ${ }^{\circledR}$ mapping catheter.

Clinical studies have shown a high AF-termination rate with reduced RF-time compared with the stepwise approach and favorable follow-up results of driver-based ablation of persistent $\mathrm{AF}[88,90]$.

The system utilizes the maximum $\mathrm{dv} / \mathrm{dt}$ [91] and therefore was originally used for the study of ventricular activation [92]. Evolution of the technology has allowed mapping of atrial tachycardia (AT) [93], paroxysmal and persistent AF [85,94-96]. Studies of persistent AF using this technology are limited to a single center experience in Bordeaux and one multi-center (AFACART study) [90]. In the multicenter AFACART study, driver only ablation resulted in termination of $\mathrm{AF}$ in $64 \%$ of those studied, and at 1-year follow-up, $78 \%$ were off antiarrhythmic drugs and $77 \%$ were free of AF recurrence. However, of those with no AF recurrence, $49 \%$ experienced at least one episode of AT [90].

Limitations noted of the Cardio-Insight system are the detection of false rotors due to the phase-based analysis; some of the AF circuits may not be displayed due to the subtraction of long R-R interval sequences only; and small signals $<0.15 \mathrm{mv}$ can be difficult to resolve from far-field signals [88,97]. Second, the assessment of the drivers within the interatrial septum may be difficult to map by the technology. Third, the processed phased data (identified driver areas) are projected on a 3D shell acquired by computed tomography rather than the atrial geometry constructed by a separate $3 \mathrm{D}$ mapping system. This may influence the ability of the operator to accurately navigate the ablation catheter to the identified sites.

\section{Novel noncontact dipole density mapping}

The AcQMap ${ }^{\circledR}$ High Resolution Imaging and Mapping System (Acutus Medical, CA, USA) provides a suite of static and dynamic 3D maps of electrical activation across an ultrasound-acquired cardiac chamber surface and localizes auxiliary electrode catheters within and around the surface. The system is comprised of an invasive diagnostic recording catheter that is inserted transvenously into either the left or right atrial chambers [98]. The catheter is attached to the AcQMap Console, which contains electronic instrumentation that drives transmission and acquisition of the ultrasound, localization and cardiac electrical data. Body-surface ECG and patch electrodes are placed on the patient to provide ECG signals and localization data to the system, respectively. A separate quadripolar catheter is transvenously placed below the diaphragm and serves as an universal electrical reference.

The AcQMap catheter is a 10F, nondeflectable catheter that is introduced into the chamber of interest over a 0.032 -inch guidewire. The distal end of the catheter is deployed into a $25 \mathrm{~mm}$ diameter spheroid, formed by six splines. Each spline has eight ultrasound transducers interspersed between eight biopotential electrodes, resulting in a total of 48 sensors of each type (Figure 1). The catheter and system are designed to acquire data without the need to contact the chamber surface, which is referred to as 'noncontact' mapping.

Ultrasound is used to image the endocardial surface by acquiring points that reconstruct the $3 \mathrm{D}$ chamber anatomy. When ultrasound is activated, acoustic waves travel through the blood until they reach the tissue surface. A portion of the wave continues travelling through the cardiac tissue while a portion is reflected and detected by the transducer. The duration of time for the acoustic wave to travel from the transducer to the cardiac surface and return to the transducer is proportional to the distance travelled. Accordingly, the system places points along the chamber wall at the calculated distance corresponding to the location of each point of reflection (Figure 2). During ultrasound 


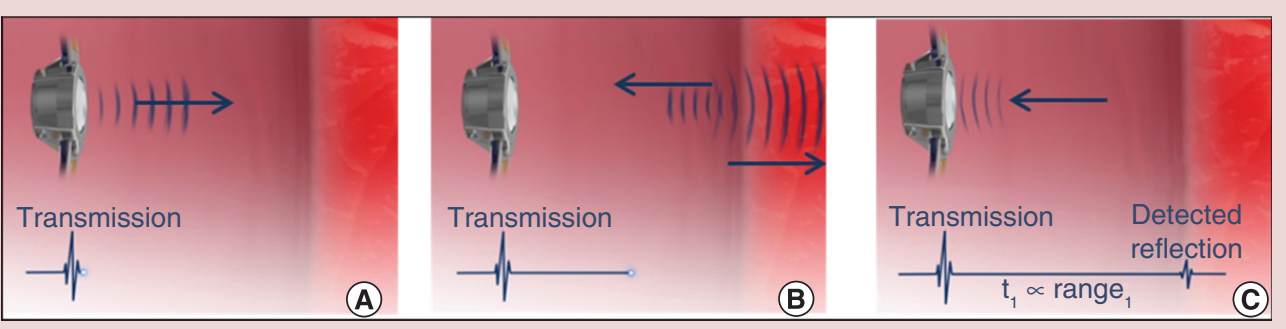

Figure 2. The process to illustrate how ultrasound transducer collects acoustic wave data. (A) Shows an acoustic pressure wave transmitted from the ultrasound transducer. (B) Demonstrates the wave propagates through the blood until it contacts the tissue. A portion of the wave continues through the tissue and a portion is reflected. (C) Shows the reflected wave detected by the ultrasound transducer. The time interval between transmission and reception of the reflection is proportional to the range of the target.

point acquisition the user continuously rotates the catheter approximately 60 degrees in both directions to ensure the entire endocardial surface is sampled. The system samples up to 115,000 surface points per minute. The entire set of surface points are usually collected in $2-3 \mathrm{~min}$. The $3 \mathrm{D}$ surface is algorithmically reconstructed from the ultrasound point-set, comprising a mesh of more than 7000 triangles. Minimal postprocessing is performed to remove unneeded points and add definition to anatomical structures. The resulting chamber anatomy corresponds to the end-diastolic size and shape. The final postprocessed anatomy is a key input into the inverse solution used to derive the location of charge sources on the endocardial surface (Supplementary Video 1 of anatomy construction).

The intracardiac potential field is measured by the 48 biopotential electrodes, which are engineered to provide low-noise and high-fidelity input into an inverse solution. Raw, noncontact and unipolar intracardiac potentials are measured across the endocardial surface by placing the catheter into the center of the chamber and recording data for any selected duration of time, as appropriate for the rhythm to be mapped. The system samples the whole potential field at a rate of 150,000 samples/s. After data are recorded, the traces from the 48 electrodes are reviewed all at once and outliers are excluded. In the case of irregular rhythms, particularly AF, an algorithm is applied to remove the QRS complex and enable continuous display of the activation wave front. Maps can be displayed as either dipole density- or voltage-based representations of the activation wave front (Supplementary Video 2 of the work flow of dipole density mapping).

\section{Dipole density principle}

Voltage has served as the gold standard in electrical analysis of cardiac signals for the past 120 years [99]. In contrast, dipole density represents the actual biophysics of cardiac activity and is a more localized entity that provides a more focused view into the details of cardiac activation. Dipole density mapping, expressed in units of $\mu$ Coulombs $/ \mathrm{cm}$, represents the magnitude of these sources on the endocardial surface of the chamber with a view of cardiac activity that is at least four-times sharper and narrower than voltage [100,101].

Dipole density is the distribution of actual charge-sources that embody the native resolution of electrical activity at the cellular level. A dipole consists of two oppositely charged particles separated by a very small distance. Each time a cell is stimulated, ions move across the cellular membrane through ion-selective channels. During ionic movement, a small dipolar imbalance in charge emerges in the adjoining extracellular medium. After the first cell is stimulated, ion channels in adjacent cells are 'recruited' and a wave of 'activation' spreads outward. The combined activation of these cells forms a macroscopic double-layer of dipoles that directly represents the wave front and, in turn, generates the cardiac potential field, measured in units of volts.

The fundamental difference between voltage and dipole density lies in both the averaging effect of 'spatial summation' and in the volume of space occupied by each. Spatial averaging causes the distribution of voltage to surround the charges and extend far beyond the compact, physical boundary of the charges. It also smooths out some of the localized details of the geometric shape of the wave front $[102,103]$. This explains why it is possible to measure the body surface ECG, although with significantly less spatial detail than intracardiac electrograms.

The AcQMap System applies an inverse solution to derive the dipolar charge sources (the cause of the field, measured as dipole density) that exist on the endocardium. The causal relationship between charge and potential 

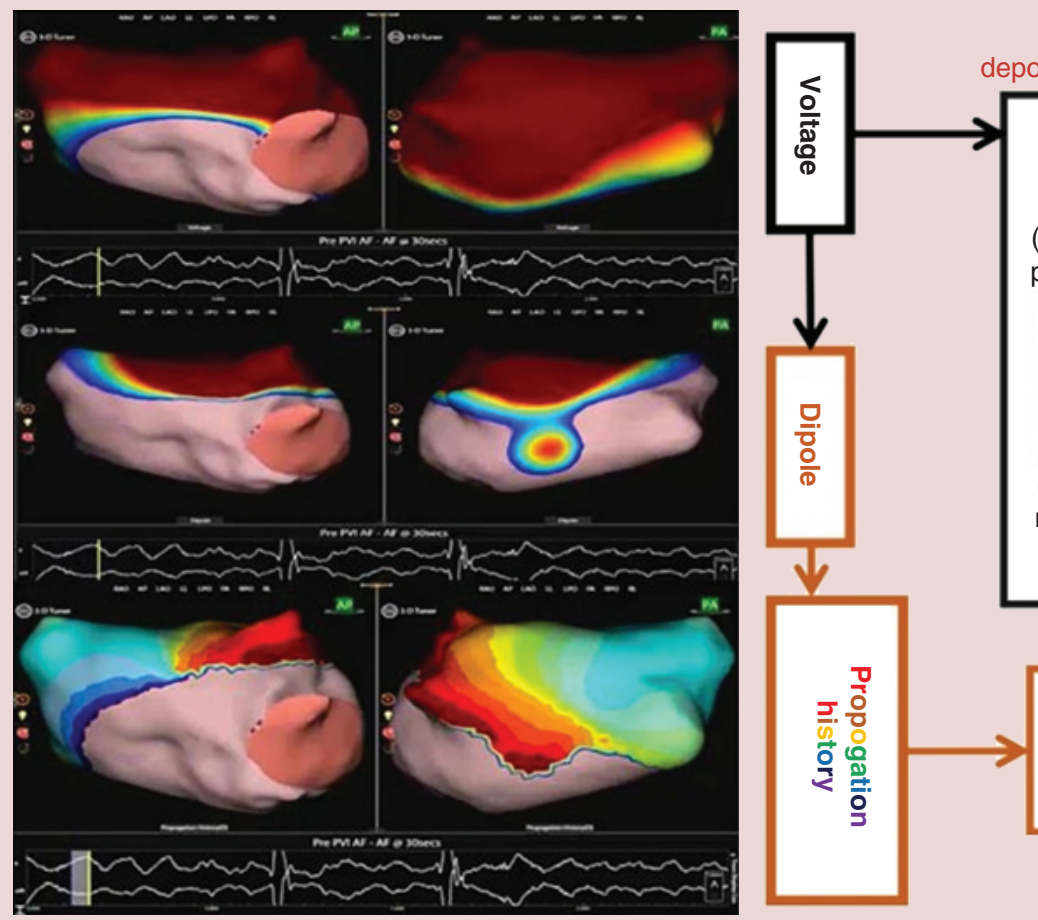

Red spot tracks [spatially derived] depolarization of the action potential (phase 2)

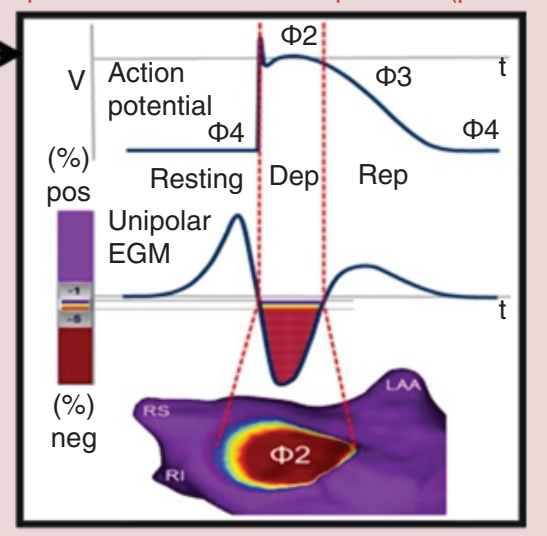

Tracks the leading edge of the depolarized wavefront (RED) and applies a [user defined] color tail.

Figure 3. Voltage map, dipole density map and propagation history map.

are based on Poisson's equation, published in 1813, which defines how the potential field at any point (measured as voltage) is equal to the local sources plus the sum of distant sources [103]. Thus, dipolar charge sources solved for by the AcQMap System are intrinsically linked to the intracavitary potentials measured.

To solve the inverse solution for dipole density, the AcQMap system leverages the fundamental constraints of cardiac activation for which charge sources exist only on and within the excitable cardiac tissue. This constraint minimizes the mathematical complexity of the inverse solution. Solving the inverse solution therefore requires three key inputs: multiple simultaneous measurements of the potential field in the chamber, which can be collected by the 48 electrodes on the AcQMap catheter; an accurate anatomic surface (ultrasound anatomy construction) used to define the location of the charge sources; and a common coordinate system in which the AcQMap catheter is localized with $3 \mathrm{D}$ coordinates that correspond with the $3 \mathrm{D}$ coordinates of the reconstructed anatomical mesh. Provision of the inputs listed above enables spatially-localized and temporally-animated derivation of the dipolar charge sources on the endocardial surface. The corresponding waves of activation are displayed across the reconstructed 3D surface through time.

The activation wave front can be displayed in its rawest form as either a dipole- or voltage-based map of depolarization, with voltage forward-calculated from the derived dipole density. Additional postprocessing of the data can be applied to extract specific information from the map. A propagation-history map uses bands of color to show the location and velocity of the leading edge of the wave front over a set duration of time. The color red is used to indicate the leading edge of the wave front with the trailing color bands showing earlier locations of the wave front. The width of the color bands conveys the conduction velocity of the wave front, with wider bands indicative of fast conduction and narrow bands of slow conduction. Figure 3 shows the relationship between raw voltage-based maps, raw dipole density based maps and propagation history maps (Supplementary Video 3).

\section{AF propagation map guided by AcQMap}

The propagation history map identifies and locates the discrete and coupled mechanisms responsible for initiating and maintaining the arrhythmia. Interpretation of propagation history maps derived from recordings of AF has revealed three atrial activation patterns of interest (API), including focal, localized rotational activation (spiraling around a small confined zone $\geq 270^{\circ}$ ) and localized irregular activation (entry/exit through, and pivoting around 


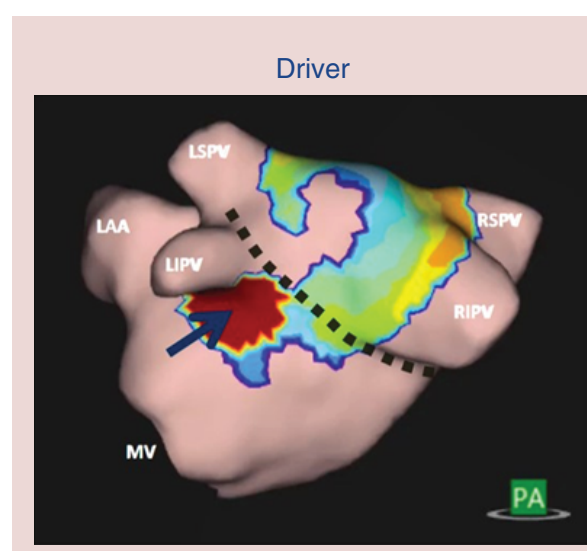

Focal

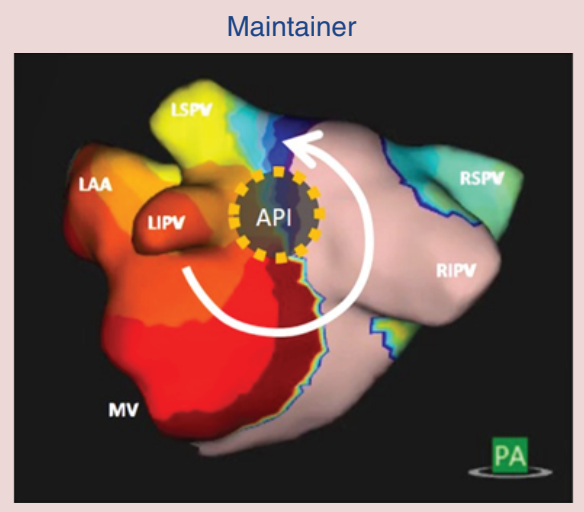

Localised rotational activation, LRA (spirals around a confined zone)
Maintainer

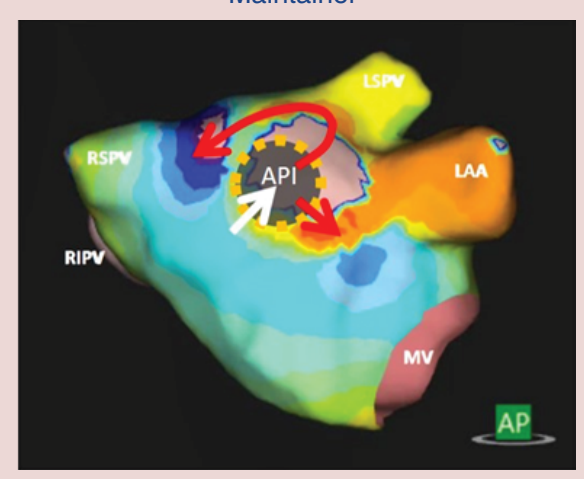

Localised irregular activation, LIA (enters and exits a confined zone)

(both with multiple directions from collision and block)

Figure 4. Three atrial activation patterns of interest identified by dipole density mapping.

a confined zone), Figure 4 and Supplementary Video 4. The areas of API are considered to be the 'driver' and/or 'maintainer' of AF. Such confined areas have dimensions ranging from 5 to $15 \mathrm{~mm}$ in diameter [104].

The ablation strategies guided by AcQMap mapping system consist PVI followed by ablation targeting these APIs (drivers and maintainers of AF) separately at each remapped stage, connecting the API guided lesion sets to near PVI circumferential lesions and/or anatomical barriers. During the procedure, a circular catheter was used to confirm the exit/entrance block as conventional PVI ablation procedure. When sinus rhythm could not be restored by ablation targeting APIs in left atrium (LA), the AcQMap catheter can be deployed in the right atrium, trying to look for APIs in right atrium. The procedural end point is AF termination by ablation [105].

The clinical experience of this combined imaging and mapping technology is still early and limited to few centers. Its limitations therefore will perhaps have to be better explored over time. In the meantime, as this platform is novel, the validation and the efficacy to guide ablation data in human is not yet available although currently being collected. The utility of the basket catheter is confined to the atrial chambers. To our knowledge, to date attempt has not been made to use this technology to map human ventricles.

\section{Live dipole density mapping of persistent AF to guide ablation: a case study}

A 54-year-old male with a 2-year history of symptomatic de novo nonvalvular persistent AF and one previous failed cardioversion underwent catheter ablation utilizing the AcQMap system. The echocardiography showed normal heart structure with mild enlargement of LA (diameter of $45 \mathrm{~mm}$ ) and normal left ventricular size and ejection fraction. After informed and consented, the patient underwent AF ablation with a strategy to achieve PVI first, followed by API targeted, non-PV drivers and maintainers ablation.

A decapolar catheter was placed in the coronary sinus and a quadripolar catheter (as the unipolar reference) was placed in the inferior vena cava. Through transeptal puncture, the AcQMap catheter was introduced into the LA over a 0.032-inch guidewire. The basket of the AcQMap catheter was fully opened and placed in the middle of the chamber. By rotating the catheter, the ultrasound crystals collect points to reconstruct the chamber anatomy. The final postprocessed anatomy was used for navigation and mapping to guide catheter ablation.

The propagation map showed two predominant APIs consisting of localized rotational activation and localized irregular activation patterns located at the mid-anterior wall and lower posterior wall near the left inferior PV (Figure 5A). Remap after PVI showed that the API at the anterior wall of the LA shifted slightly toward the right-sided PVs (Figure 5B).

The API on the anterior wall was ablated first. This cluster of ablation was then connected to the nearest electrical barrier (circumferential lesion near the right PVs) and anatomical barrier (anterior aspect of the mitral valve annulus). The remap after this lesion set revealed a significant conduction modification of the anterior API. The posterior API persisted. This was targeted with ablation next and the focal lesion set was joined to the electrical 


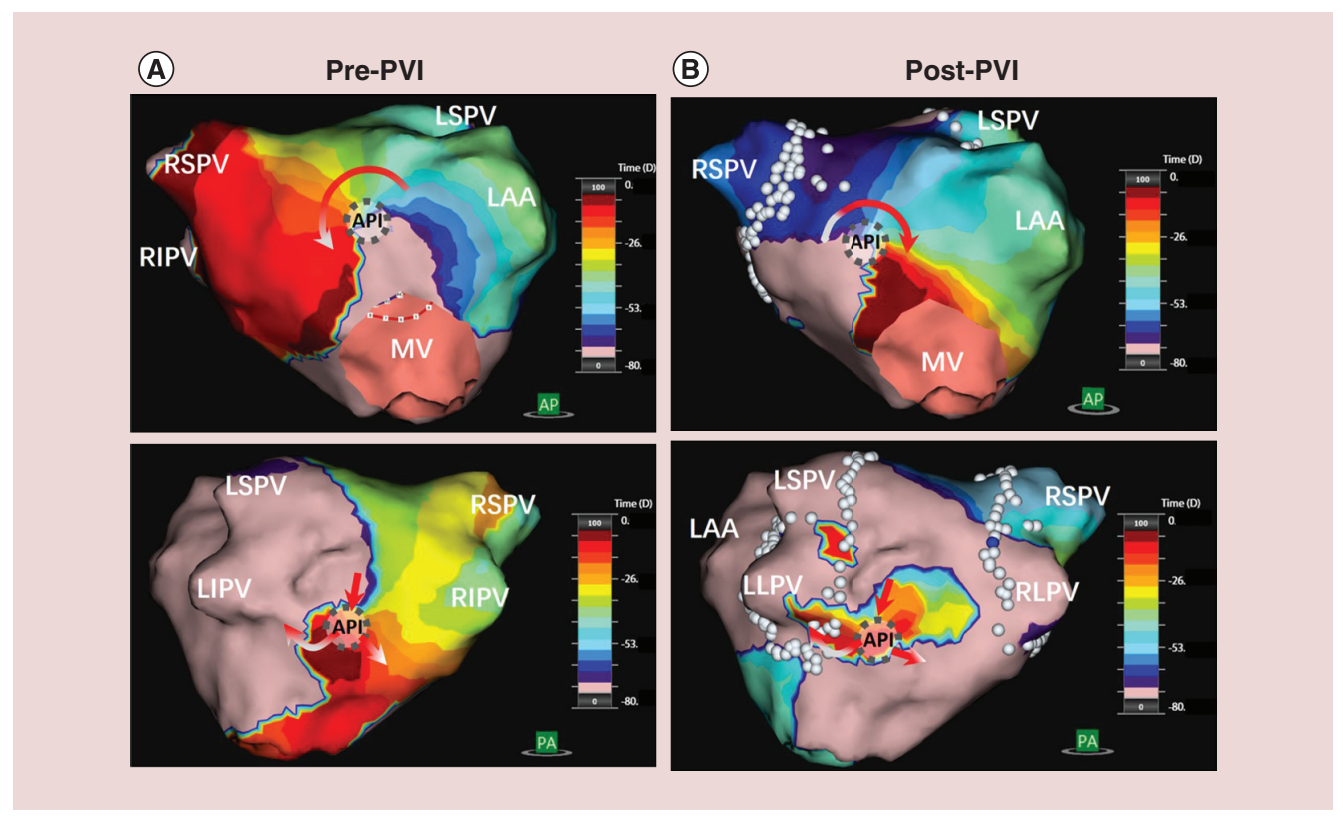

Figure 5. Initial pre- and postpulmonary vein electrical isolation propagation history map in persistent atrial fibrillation. Two spatial and temporal consistent API located on middle anterior wall (AP view) and on the lower posterior wall (PA view) pre-PVI (A) and post-PVI (B), respectively.

AP: Anterior-posterior veiw; API: Atrial activation pattern of interest; LIPV: Left inferior pulmonary vein; PA: Posterior-anterior view; PVI: Pulmonary vein electrical isolation.

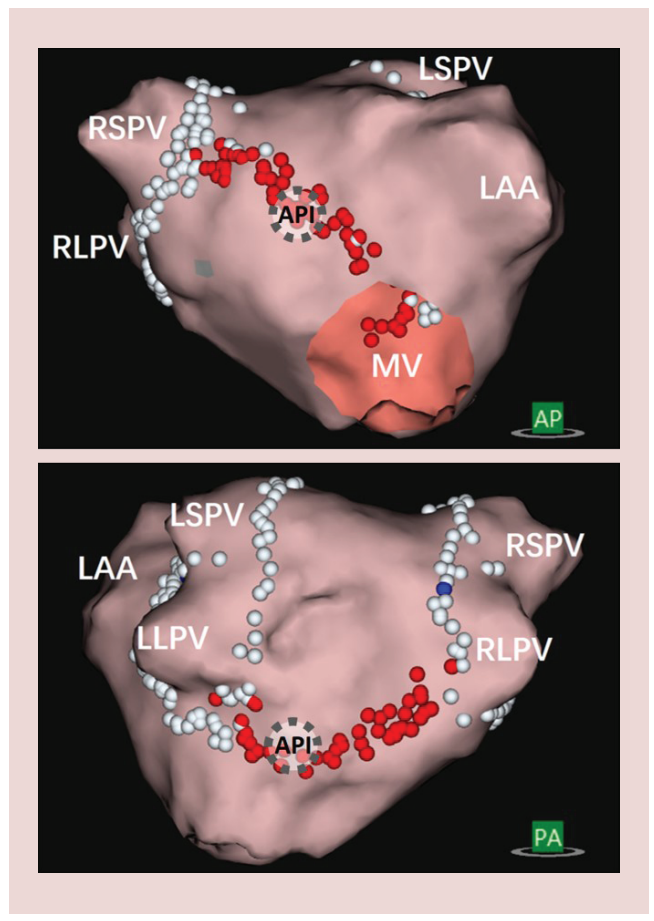

Figure 6. The ablation strategies guided by dipole density mapping. After PVI, the API at the anterior LA wall was ablated first, connecting the lesion sets to the circumferential lesion near right PV and anterior mitral valve annulus (AP view). The API at the low posterior wall was targeted next, followed by connecting that to left and right circumferential lesions posteriorly (PA view).

AP: Anterior-posterior veiw; API: Atrial activation pattern of interest; LA: Left atrial; PA: Posterior-anterior view; PV: Pulmonary vein; PVI: Pulmonary vein electrical isolation.

barriers (circumferential lesions near the left/right inferior PVs), see Figure 6. On completion of the lower posterior line, AF terminated with conversion to LA tachycardia (cycle length of $215 \mathrm{~ms}$ with the coronary sinus sequence from distal to proximal). The remap of this LA tachycardia showed that it was a macrore-entrant tachycardia conducting through an isthmus in the middle of the previously ablated anterior line (Figure 7). Further ablation at this site terminated the AT to sinus rhythm (Supplementary Video 5). 


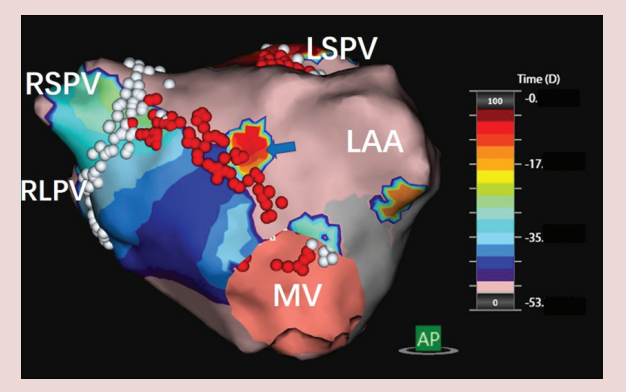

Figure 7. Atrial tachycardia dipole density map of the left atrium. A gap (blue arrow) in the middle of anterior ablation lesion was detected by the dipole density mapping during atrial tachycardia (AT; cycle length of $215 \mathrm{~ms}$ ). Ablation at the gap terminated the AT to sinus rhythm.

AP: Anterior-posterior veiw.

\title{
Ongoing studies in dipole density mapping guided ablation
}

The novel noncontact AcQMap mapping system may represent a significant advance in treating persistent AF when compared with conventional 3D mapping systems, but clinical data of AF ablation in assessing this technology are at a preliminary stage. The prospective, single-arm, international multicenter and nonrandomized UNCOVER-AF clinical study (NCT02825992) being conducted at the moment aimed to address both procedural and long-term outcomes of catheter ablation in 125 persistent AF patients guided by this novel mapping system. Large multicenter randomized control trial is required to assess the efficacy of dipole density mapping guided ablation to treat $\mathrm{AF}$.

\section{Future perspective}

In the last decade, significant efforts and intense research have been incorporated into newer mapping systems to improve procedural efficiency and outcomes for AF ablation. Accurate panoramic live mapping of AF is a novel approach that may lead to an increasingly successful outcome of ablation. There are several fundamental and important requisite in understanding the complexity of persistent $\mathrm{AF}$, so clinicians can target optimal ablation sites to treat persistent $\mathrm{AF}$. They include an accurate anatomic surface mesh and an accurate live mapping of the dynamic rapidly changing wave fronts. Identification and understanding of fibrillary circuits is a formidable challenge but durable success for ablation also requires effective ablation lesion delivery to achieve transmural and durable lesions in the targeted location.

The next phase in AF mapping will depend heavily on advancing fundamental knowledge of arrhythmia mechanisms in humans. Increasing success will depend upon the ability to simultaneously accurately map temporal, spatial and high-resolution electrogram data to offer bespoke lesions to target both non-PV triggered and complex persistent $\mathrm{AF}$.

\section{Conclusion}

Persistent AF is a complex, patient-specific arrhythmia. The result of catheter ablation to treat this arrhythmia is currently suboptimal, although PVI remains the cornerstone of the ablation strategy. Conventional contact and noncontact voltage-based mapping may not adequately identify patient-specific drivers and/or maintainers of AF due to their spatially averaged representation of the actual complex and irregular spatiotemporal characteristics of cardiac conduction. The emergence of simultaneous global chamber, live mapping systems may help us to accurately identify clinically critical non-PV targets for ablation with an aim to improve clinical outcome. Future clinical studies are required to investigate if these novel mapping technologies such as noncontact dipole density mapping can provide a better understanding of the mechanistic characterization of AF and individualized ablation strategies that relate to an improved clinical outcome.

\author{
Supplementary data \\ To view the supplementary data that accompany this paper, please visit the journal website at: See online at: \\ www.futuremedicine.com/doi/suppl/10.2217/fca-2017-0109
}

Financial \& competing interests disclosure

The authors have no relevant affiliations or financial involvement with any organization or entity with a financial interest in or financial conflict with the subject matter or materials discussed in the manuscript. This includes employment, consultancies, honoraria, stock ownership or options, expert testimony, grants or patents received or pending, or royalties. 
No writing assistance was utilized in the production of this manuscript.

Executive summary

\section{Background}

- Atrial fibrillation (AF) is the most common clinical arrhythmia encountered. Catheter ablation has become the first-line therapy for symptomatic drug-refractory paroxysmal and persistent AF.

Evolution of AF ablation \& the challenges in ablation of persistent AF

- Currently, pulmonary vein isolation is the cornerstone of ablation for both paroxysmal and persistent AF. Adjunctive ablation strategies in addition to pulmonary vein electrical isolation have been investigated, but the medium to longer term follow-up clinical outcomes were suboptimal.

- Understanding patient-specific AF mechanisms is challenging.

- Live global electroanatomic mapping allows us to gain insights to triggers and maintainers of AF which can be targeted to improve ablation outcome.

Noncontact endocardial mapping with EnSite ${ }^{\circledR}$ Array

- The first noncontact, global chamber mapping technology use 64 unipolar electrodes to record cavitary virtual unipolar electrograms and display these calculated electrograms on a surface mesh.

Contact focal impulse and rotor modulation phase mapping

- The technology identified sites of spiral rotors and focal drivers of AF in humans for the first time by using a 64-electrode, contact mapping basket catheter to collect electrophysiologic activity.

Noncontact body surface mapping

- A noninvasive mapping system that uses 252 external electrodes in combination with computed tomography to record bi-atrial unipolar electrograms and create simultaneous bi-atrial 3D maps.

Novel noncontact dipole density mapping

- The AcQMap ${ }^{\circledR}$ catheter is a basket catheter with ultrasound transducers interspersed between biopotential electrodes. Ultrasound is used to image and reconstruct the endocardial surface. The intracardiac potential field is measured by the biopotential electrodes.

- Dipole density represents the actual biophysics of cardiac activity and is a more localized entity that provides a more focused view into the details of cardiac activation.

Future perspective

- Significant efforts and intense research have developed newer mapping systems to improve accuracy and efficiency of electroanatomic mapping. Accurate panoramic live mapping of $A F$ is a novel approach that may lead to an increasingly successful outcome of ablation.

- Improved outcome from persistent AF ablation will depend upon the ability of the mapping system to simultaneously and accurately map temporal, spatial and high-resolution electrogram data to offer bespoke lesions to target nonpulmonary vein triggered complex persistent AF.

\section{References}

Papers of special note have been highlighted as: $\bullet$ of interest; $\bullet \bullet$ of considerable interest

1 Go AS, Hylek EM, Phillips KA et al. Prevalence of diagnosed atrial fibrillation in adults: national implications for rhythm management and stroke prevention: the AnTicoagulation and Risk Factors in Atrial Fibrillation (ATRIA) Study. JAMA 285(18), 2370-2375 (2001).

2 Wolf PA, Benjamin EJ, Belanger AJ, Kannel WB, Levy D, D'Agostino RB. Secular trends in the prevalence of atrial fibrillation: the Framingham Study. Am. Heart J. 131(4), 790-795 (1996).

3 Chugh SS, Havmoeller R, Narayanan K et al. Worldwide epidemiology of atrial fibrillation: a Global Burden of Disease 2010 Study. Circulation 129(8), 837-847 (2014).

4 Rahman F, Kwan GF, Benjamin EJ. Global epidemiology of atrial fibrillation. Nat. Rev. Cardiol. 11(11), 639-654 (2014).

- We can understand the epidemiology of atrial fibrillation (AF) in the whole world by this paper.

5 Heeringa J, Van Der Kuip DA, Hofman A et al. Prevalence, incidence and lifetime risk of atrial fibrillation: the Rotterdam study. Eur. Heart J. 27(8), 949-953 (2006).

6 Ball J, Carrington MJ, McMurray JJ, Stewart S. Atrial fibrillation: profile and burden of an evolving epidemic in the 21 st century. Int. J. Cardiol. 167(5), 1807-1824 (2013).

7 Zoni-Berisso M, Filippi A, Landolina $\mathrm{M}$ et al. Frequency, patient characteristics, treatment strategies, and resource usage of atrial fibrillation (from the Italian Survey of Atrial Fibrillation Management [ISAF] study). Am. J. Cardiol. 111(5), 705-711 (2013).

8 Meinertz T, Kirch W, Rosin L, Pittrow D, Willich SN, Kirchhof P. Management of atrial fibrillation by primary care physicians in Germany: baseline results of the ATRIUM registry. Clin. Res. Cardiol. 100(10), 897-905 (2011).

9 Nabauer M, Gerth A, Limbourg T et al. The Registry of the German Competence NETwork on Atrial Fibrillation: patient characteristics and initial management. Europace 11(4), 423-434 (2009). 
10 Nieuwlaat R, Capucci A, Camm AJ et al. Atrial fibrillation management: a prospective survey in ESC member countries: the Euro Heart Survey on Atrial Fibrillation. Eur. Heart J. 26(22), 2422-2434 (2005).

11 Di Pasquale G, Mathieu G, Maggioni AP et al. Current presentation and management of 7148 patients with atrial fibrillation in cardiology and internal medicine hospital centers: the ATA AF study. Int. J. Cardiol. 167(6), 2895-2903 (2013).

12 Kannel WB, Wolf PA, Benjamin EJ, Levy D. Prevalence, incidence, prognosis, and predisposing conditions for atrial fibrillation: population-based estimates. Am. J. Cardiol. 82(8a), 2n-9n (1998).

13 Jarman JW, Hunter TD, Hussain W, March JL, Wong T, Markides V. Stroke rates before and after ablation of atrial fibrillation and in propensity-matched controls in the UK. Pragmat. Obs. Res. 8, 107-118 (2017).

14 Wang TJ, Larson MG, Levy D et al. Temporal relations of atrial fibrillation and congestive heart failure and their joint influence on mortality: the Framingham Heart Study. Circulation 107(23), 2920-2925 (2003).

15 Miyasaka Y, Barnes ME, Bailey KR et al. Mortality trends in patients diagnosed with first atrial fibrillation: a 21-year community-based study. J. Am. Coll. Cardiol. 49(9), 986-992 (2007).

16 Jacobs V, Cutler MJ, Day JD, Bunch TJ. Atrial fibrillation and dementia. Trends Cardiovasc. Med. 25(1), 44-51 (2015).

17 Aldrugh S, Sardana M, Henninger N, Saczynski JS, McManus DD. Atrial fibrillation, cognition and dementia: a review. J. Cardiovasc. Electrophysiol. 28(8), 958-965 (2017).

18 Chen LY, Benditt DG, Alonso A. Atrial fibrillation and its association with sudden cardiac death. Circ. J. 78(11), 2588-2593 (2014)

19 Chen LY, Sotoodehnia N, Buzkova P et al. Atrial fibrillation and the risk of sudden cardiac death: the atherosclerosis risk in communities study and cardiovascular health study. JAMA Int. Med. 173(1), 29-35 (2013).

20 Camm AJ, Lip GY, De Caterina R et al. 2012 focused update of the ESC Guidelines for the management of atrial fibrillation: an update of the 2010 ESC Guidelines for the management of atrial fibrillation. Developed with the special contribution of the European Heart Rhythm Association. Eur. Heart J. 33(21), 2719-2747 (2012).

21 January CT, Wann LS, Alpert JS et al. 2014 AHA/ACC/HRS guideline for the management of patients with atrial fibrillation: a report of the American College of Cardiology/American Heart Association Task Force on Practice Guidelines and the Heart Rhythm Society. J. Am. Coll. Cardiol. 64(21), e1-e76 (2014).

- Current guideline of AF management.

22 Calkins H, Hindricks G, Cappato R et al. 2017 HRS/EHRA/ECAS/APHRS/SOLAECE expert consensus statement on catheter and surgical ablation of atrial fibrillation. Heart Rhythm 14(10), e275-e444 (2017).

23 Wazni OM, Marrouche NF, Martin DO et al. Radiofrequency ablation vs antiarrhythmic drugs as first-line treatment of symptomatic atrial fibrillation: a randomized trial. JAMA 293(21), 2634-2640 (2005).

24 Cosedis Nielsen J, Johannessen A, Raatikainen P et al. Radiofrequency ablation as initial therapy in paroxysmal atrial fibrillation. $N$. Engl. J. Med. 367(17), 1587-1595 (2012).

25 Morillo CA, Verma A, Connolly SJ et al. Radiofrequency ablation vs antiarrhythmic drugs as first-line treatment of paroxysmal atrial fibrillation (RAAFT-2): a randomized trial. JAMA 311(7), 692-700 (2014).

26 Jones DG, Haldar SK, Hussain W et al. A randomized trial to assess catheter ablation versus rate control in the management of persistent atrial fibrillation in heart failure. J. Am. Coll. Cardiol. 61(18), 1894-1903 (2013).

- Typical paper that shows catheter ablation of AF is superior to medication, particularly for heart failure patients.

27 Jalife J, Berenfeld O, Mansour M. Mother rotors and fibrillatory conduction: a mechanism of atrial fibrillation. Cardiovasc. Res. 54(2), 204-216 (2002).

28 Schotten U, Verheule S, Kirchhof P, Goette A. Pathophysiological mechanisms of atrial fibrillation: a translational appraisal. Physiol. Rev. 91(1), 265-325 (2011).

29 Nattel S. New ideas about atrial fibrillation 50 years on. Nature 415(6868), 219-226 (2002).

30 Kalifa J, Jalife J, Zaitsev AV et al. Intra-atrial pressure increases rate and organization of waves emanating from the superior pulmonary veins during atrial fibrillation. Circulation 108(6), 668-671 (2003).

31 Haissaguerre M, Jais $\mathrm{P}$, Shah DC et al. Spontaneous initiation of atrial fibrillation by ectopic beats originating in the pulmonary veins. $N$. Engl. J. Med. 339(10), 659-666 (1998).

32 Jais $\mathrm{P}$, Haissaguerre M, Shah DC et al. A focal source of atrial fibrillation treated by discrete radiofrequency ablation. Circulation 95(3), 572-576 (1997).

33 Oral H, Knight BP, Tada $\mathrm{H}$ et al. Pulmonary vein isolation for paroxysmal and persistent atrial fibrillation. Circulation 105(9), 1077-1081 (2002).

34 Oral H, Pappone C, Chugh A et al. Circumferential pulmonary-vein ablation for chronic atrial fibrillation. N. Engl. J. Med. 354(9), 934-941 (2006).

35 Forleo GB, Mantica M, De Luca L et al. Catheter ablation of atrial fibrillation in patients with diabetes mellitus Type 2: results from a randomized study comparing pulmonary vein isolation versus antiarrhythmic drug therapy. J. Cardiovasc. Electrophysiol. 20(1), 22-28 (2009). 
36 Wynn GJ, Das M, Bonnett LJ, Panikker S, Wong T, Gupta D. Efficacy of catheter ablation for persistent atrial fibrillation: a systematic review and meta-analysis of evidence from randomized and nonrandomized controlled trials. Circ. Arrhythm. Electrophysiol. 7(5), $841-852$ (2014).

37 Nademanee K, McKenzie J, Kosar E et al. A new approach for catheter ablation of atrial fibrillation: mapping of the electrophysiologic substrate. J. Am. Coll. Cardiol. 43(11), 2044-2053 (2004).

38 Brooks AG, Stiles MK, Laborderie J et al. Outcomes of long-standing persistent atrial fibrillation ablation: a systematic review. Heart Rhythm 7(6), 835-846 (2010).

39 Zhang Z, Letsas KP, Zhang $\mathrm{N}$ et al. Linear ablation following pulmonary vein isolation in patients with atrial fibrillation: a meta-analysis. Pacing Clin. Electrophysiol. 39(6), 623-630 (2016).

40 Yao Y, Zheng L, Zhang S et al. Stepwise linear approach to catheter ablation of atrial fibrillation. Heart Rhythm 4(12), 1497-1504 (2007).

41 Knecht $\mathrm{S}$, Hocini M, Wright $\mathrm{M}$ et al. Left atrial linear lesions are required for successful treatment of persistent atrial fibrillation. Eur. Heart J. 29(19), 2359-2366 (2008).

42 Wynn GJ, Panikker S, Morgan M et al. Biatrial linear ablation in sustained nonpermanent AF: results of the substrate modification with ablation and antiarrhythmic drugs in nonpermanent atrial fibrillation (SMAN-PAF) trial. Heart Rhythm 13(2), 399-406 (2016).

43 Ghias M, Scherlag BJ, Lu Z et al. The role of ganglionated plexi in apnea-related atrial fibrillation. J. Am. Coll. Cardiol. 54(22), 2075-2083 (2009).

$44 \mathrm{Lu}$ Z, Scherlag BJ, Lin J et al. Autonomic mechanism for initiation of rapid firing from atria and pulmonary veins: evidence by ablation of ganglionated plexi. Cardiovasc. Res. 84(2), 245-252 (2009).

45 Pokushalov E, Romanov A, Artyomenko S et al. Ganglionated plexi ablation for longstanding persistent atrial fibrillation. Europace 12(3), 342-346 (2010).

46 Lin WS, Tai CT, Hsieh MH et al. Catheter ablation of paroxysmal atrial fibrillation initiated by non-pulmonary vein ectopy. Circulation 107(25), 3176-3183 (2003).

47 Di Biase L, Burkhardt JD, Mohanty P et al. Left atrial appendage: an underrecognized trigger site of atrial fibrillation. Circulation 122(2), 109-118 (2010).

48 Kurotobi $\mathrm{T}$, Ito $\mathrm{H}$, Inoue $\mathrm{K}$ et al. Marshall vein as arrhythmogenic source in patients with atrial fibrillation: correlation between its anatomy and electrophysiological findings. J. Cardiovasc. Electrophysiol. 17(10), 1062-1067 (2006).

49 Panikker S, Jarman JW, Virmani R et al. Left atrial appendage electrical isolation and concomitant device occlusion to treat persistent atrial fibrillation: a first-in-human safety, feasibility, and efficacy study. Circ. Arrhythm. Electrophysiol. 9(7), pii:e003710 (2016).

50 Di Biase L, Burkhardt JD, Mohanty P et al. Left atrial appendage isolation in patients with longstanding persistent AF undergoing catheter ablation: BELIEF trial. J. Am. Coll. Cardiol. 68(18), 1929-1940 (2016).

51 Scherr D, Khairy P, Miyazaki S et al. Five-year outcome of catheter ablation of persistent atrial fibrillation using termination of atrial fibrillation as a procedural endpoint. Circ. Arrhythm. Electrophysiol. 8(1), 18-24 (2015).

52 O'Neill MD, Wright M, Knecht $\mathrm{S}$ et al. Long-term follow-up of persistent atrial fibrillation ablation using termination as a procedural endpoint. Eur. Heart J. 30(9), 1105-1112 (2009).

53 Haldar SK, Jones DG, Bahrami T et al. Catheter ablation vs electrophysiologically guided thoracoscopic surgical ablation in long-standing persistent atrial fibrillation: the CASA-AF study. Heart Rhythm 14(11), 1596-1603 (2017).

54 Weerasooriya R, Shah AJ, Hocini M, Jais P, Haissaguerre M. Contemporary challenges of catheter ablation for atrial fibrillation. Clin. Ther. 36(9), 1145-1150 (2014).

55 Shah AJ, Liu X, Jadidi AS, Haissaguerre M. Early management of atrial fibrillation: from imaging to drugs to ablation. Nat. Rev. Cardiol. 7(6), 345-354 (2010).

56 Haldar SK, Jones DG, Khan H et al. Characterising the difference in electrophysiological substrate and outcomes between heart failure and non-heart failure patients with persistent atrial fibrillation. Europace doi:10.1093/europace/euw380 (2017).

57 Jarman JW, Wong T, Kojodjojo P et al. Organizational index mapping to identify focal sources during persistent atrial fibrillation. J. Cardiovasc. Electrophysiol. 25(4), 355-363 (2014).

58 Farrell M, Yoneda Z, Montgomery J et al. Non-pulmonary vein mediated atrial fibrillation: a novel sub-phenotype. PLoS ONE 12(9), e0184354 (2017).

59 Chen SA, Tai CT. Catheter ablation of atrial fibrillation originating from the non-pulmonary vein foci. J. Cardiovasc. Electrophysiol. 16(2), 229-232 (2005).

60 Hwang C, Wu TJ, Doshi RN, Peter CT, Chen PS. Vein of marshall cannulation for the analysis of electrical activity in patients with focal atrial fibrillation. Circulation 101(13), 1503-1505 (2000).

61 Corradi D, Callegari S, Gelsomino S, Lorusso R, Macchi E. Morphology and pathophysiology of target anatomical sites for ablation procedures in patients with atrial fibrillation: part II: pulmonary veins, caval veins, ganglionated plexi, and ligament of Marshall. Int. J. Cardiol. 168(3), 1769-1778 (2013). 
62 Panikker S, Virmani R, Sakakura K et al. Left atrial appendage electrical isolation and concomitant device occlusion: a safety and feasibility study with histologic characterization. Heart Rhythm 12(1), 202-210 (2015).

63 Jarman JW, Panikker S, Das M et al. Relationship between contact force sensing technology and medium-term outcome of atrial fibrillation ablation: a multicenter study of 600 patients. J. Cardiovasc. Electrophysiol. 26(4), 378-384 (2015).

64 Ullah W, Hunter RJ, Haldar S et al. Comparison of robotic and manual persistent AF ablation using catheter contact force sensing: an international multicenter registry study. Pacing Clin. Electrophysiol. 37(11), 1427-1435 (2014).

65 Reddy VY, Dukkipati SR, Neuzil P et al. Randomized, controlled trial of the safety and effectiveness of a contact force-sensing irrigated catheter for ablation of paroxysmal atrial fibrillation: results of the TactiCath contact force ablation catheter study for atrial fibrillation (TOCCASTAR) study. Circulation 132(10), 907-915 (2015).

66 De Vries LJ, Szili-Torok T. Optimizing contact force during ablation of atrial fibrillation: available technologies and a look to the future. Future Cardiol. 12(2), 197-207 (2016).

67 Conti S, Weerasooriya R, Novak P et al. Contact force sensing for ablation of persistent atrial fibrillation: a randomized, multicenter trial. Heart Rhythm doi:10.1016/j.hrthm.2017.10.010 (2017).

68 Huynh K. Atrial fibrillation. Adenosine testing during catheter ablation of AF reduces recurrent atrial tachyarrhythmia. Nat. Rev. Cardiol. 12(10), $562(2015)$.

69 McLellan AJA, Kumar S, Smith C et al. The role of adenosine challenge in catheter ablation for atrial fibrillation: a systematic review and meta-analysis. Int. J. Cardiol. 236, 253-261 (2017).

70 Miyazaki S, Kobori A, Hocini M et al. Clinical utility of adenosine-infusion test at a repeat atrial fibrillation ablation procedure. Heart Rhythm 10(5), 629-635 (2013).

71 Andrade JG, Pollak SJ, Monir G et al. Pulmonary vein isolation using a pace-capture-guided versus an adenosine-guided approach: effect on dormant conduction and long-term freedom from recurrent atrial fibrillation - a prospective study. Circ. Arrhythm. Electrophysiol. 6(6), 1103-1108 (2013).

72 Hindricks G, Kottkamp H. Simultaneous noncontact mapping of left atrium in patients with paroxysmal atrial fibrillation. Circulation 104(3), 297-303 (2001).

73 Earley MJ, Abrams DJ, Sporton SC, Schilling RJ. Validation of the noncontact mapping system in the left atrium during permanent atrial fibrillation and sinus rhythm. J. Am. Coll. Cardiol. 48(3), 485-491 (2006).

74 Kumagai K, Nakashima H. Noncontact mapping-guided catheter ablation of atrial fibrillation. Circ. J. 73(2), 233-241 (2009).

75 Narayan SM, Krummen DE, Rappel WJ. Clinical mapping approach to diagnose electrical rotors and focal impulse sources for human atrial fibrillation. J. Cardiovasc. Electrophysiol. 23(5), 447-454 (2012).

76 Narayan SM, Krummen DE, Shivkumar K, Clopton P, Rappel WJ, Miller JM. Treatment of atrial fibrillation by the ablation of localized sources: CONFIRM (Conventional Ablation for Atrial Fibrillation With or Without Focal Impulse and Rotor Modulation) trial. J. Am. Coll. Cardiol. 60(7), 628-636 (2012).

-. Shows the detail of AF with focal impulse and rotor modulation.

77 Narayan SM, Krummen DE, Clopton P, Shivkumar K, Miller JM. Direct or coincidental elimination of stable rotors or focal sources may explain successful atrial fibrillation ablation: on-treatment analysis of the CONFIRM trial (conventional ablation for AF with or without focal impulse and rotor modulation). J. Am. Coll. Cardiol. 62(2), 138-147 (2013).

78 Narayan SM, Baykaner T, Clopton P et al. Ablation of rotor and focal sources reduces late recurrence of atrial fibrillation compared with trigger ablation alone: extended follow-up of the CONFIRM trial (Conventional Ablation for Atrial Fibrillation With or Without Focal Impulse and Rotor Modulation). J. Am. Coll. Cardiol. 63(17), 1761-1768 (2014).

79 Benharash P, Buch E, Frank P et al. Quantitative analysis of localized sources identified by focal impulse and rotor modulation mapping in atrial fibrillation. Circ. Arrhythm. Electrophysiol. 8(3), 554-561 (2015).

80 Buch E, Share M, Tung R et al. Long-term clinical outcomes of focal impulse and rotor modulation for treatment of atrial fibrillation: a multicenter experience. Heart Rhythm 13(3), 636-641 (2016).

81 Gianni C, Mohanty S, Di Biase L et al. Acute and early outcomes of focal impulse and rotor modulation (FIRM)-guided rotors-only ablation in patients with nonparoxysmal atrial fibrillation. Heart Rhythm 13(4), 830-835 (2016).

82 Miller JM, Kalra V, Das MK et al. Clinical benefit of ablating localized sources for human atrial fibrillation: the Indiana University FIRM registry. J. Am. Coll. Cardiol. 69(10), 1247-1256 (2017).

83 Miller JM, Kowal RC, Swarup V et al. Initial independent outcomes from focal impulse and rotor modulation ablation for atrial fibrillation: multicenter FIRM registry. J. Cardiovasc. Electrophysiol. 25(9), 921-929 (2014).

84 Zaman JAB, Schricker A, Lalani GG, Trikha R, Krummen DE, Narayan SM. Focal impulse and rotor mapping (FIRM): conceptualizing and treating atrial fibrillation. J. Atr. Fibrillation 7(2), 1103 (2014).

85 Haissaguerre M, Hocini M, Shah AJ et al. Noninvasive panoramic mapping of human atrial fibrillation mechanisms: a feasibility report. J. Cardiovasc. Electrophysiol. 24(6), 711-717 (2013). 
86 Ramanathan C, Ghanem RN, Jia P, Ryu K, Rudy Y. Noninvasive electrocardiographic imaging for cardiac electrophysiology and arrhythmia. Nat. Med. 10(4), 422-428 (2004).

87 Lim HS, Sacher F, Zellerhoff S et al. Persistent atrial fibrillation ablation: conventional versus driver-guided strategy. Future. Cardiol. 11(6), 697-703 (2015).

88 Haissaguerre M, Hocini M, Denis A et al. Driver domains in persistent atrial fibrillation. Circulation 130(7), 530-538 (2014).

-• Clinical study of AF using noncontact body surface mapping.

89 Cuculich PS, Wang Y, Lindsay BD et al. Noninvasive characterization of epicardial activation in humans with diverse atrial fibrillation patterns. Circulation 122(14), 1364-1372 (2010).

90 Knecht S, Sohal M, Deisenhofer I et al. Multicentre evaluation of non-invasive biatrial mapping for persistent atrial fibrillation ablation: the AFACART study. Europace 19(8), 1302-1309 (2017).

91 Rudy Y. Noninvasive electrocardiographic imaging of arrhythmogenic substrates in humans. Circ. Res. 112(5), 863-874 (2013).

92 Oster HS, Taccardi B, Lux RL, Ershler PR, Rudy Y. Noninvasive electrocardiographic imaging: reconstruction of epicardial potentials, electrograms, and isochrones and localization of single and multiple electrocardiac events. Circulation 96(3), 1012-1024 (1997).

93 Wang Y, Cuculich PS, Woodard PK, Lindsay BD, Rudy Y. Focal atrial tachycardia after pulmonary vein isolation: noninvasive mapping with electrocardiographic imaging (ECGI). Heart Rhythm 4(8), 1081-1084 (2007).

94 Shah A, Hocini M, Haissaguerre M, Jais P. Non-invasive mapping of cardiac arrhythmias. Curr. Cardiol. Rep. 17(8), 60 (2015).

95 Dubois R, Shah AJ, Hocini M et al. Non-invasive cardiac mapping in clinical practice: application to the ablation of cardiac arrhythmias. J. Electrocardiol. 48(6), 966-974 (2015).

96 Shah AJ, Hocini M, Xhaet $\mathrm{O}$ et al. Validation of novel 3-dimensional electrocardiographic mapping of atrial tachycardias by invasive mapping and ablation: a multicenter study. J. Am. Coll. Cardiol. 62(10), 889-897 (2013).

97 Yamashita S, Shah AJ, Mahida S et al. Body surface mapping to guide atrial fibrillation ablation. Arrhythm ElectroPhysiol. Rev. 4(3), 172-176 (2015).

98 Grace A, Verma A, Willems S. Dipole density mapping of atrial fibrillation. Eur. Heart J. 38(1), 5-9 (2017).

-• The first paper which illustrates AF ablation guided by dipole density mapping.

99 Waller AD. A demonstration on man of electromotive changes accompanying the heart's beat. J. Physiol. 8(5), 229-234 (1887).

100 Duytschaever M, Willems S, Tavernier R et al. Abstract S349 PO04-82: novel ultrasound and dipole density mapping: a feasibility study in patients with atrial flutter Heart Rhythm 12(Suppl. 5), PO04-PO82 (2015).

101 Heck P, Neuzil P, Reddy V, Grace A. Abstract 17111: novel global ultrasound imaging and continuous dipole density mapping: initial findings in AF patients. Circulation 132(Suppl. 3), A17111 (2015).

102 Paul J. Nahin. Oliver Heaviside: The Life, Work, And Times Of An Electrical Genius Of The Victorian Age. Johns Hopkins University Press, Baltimore, MD, USA, 108-112 (2002).

103 WS Bulletin. de la Société Philomatique de Paris, Paris, France, t.3 (1813).

104 Grace A, Heck P, Reddy V et al. Initial procedural results from the DDRAMATIC SVT Study: AF mechanism identification and localization using dipole density mapping to guide ablation strategy. Circulation 134(Suppl. 1), Abstract 17822 (2016).

105 Shi R, Eric Lim, Wajid Hussain et al. Abstract S264 C-PO03-70. First-in-man biatrial dipole density mapping of AT arising during ablation of persistent AF: an iterative strategy of electrofunctional mapping and ablation. Heart Rhythm 14(Suppl. 5), C-PO03-70 (2017). 
